\title{
Heat transfer nanofluid based on curly ultra-long multi-wall carbon nanotubes
}

\author{
Slawomir Boncel ${ }^{1} \cdot$ Aurelia Zniszczol $^{1,2}$ - Miroslawa Pawlyta ${ }^{3} \cdot$ Krzysztof Labisz $^{3}$. \\ Grzegorz Dzido ${ }^{2}$
}

Received: 22 October 2016 / Accepted: 8 August 2017 /Published online: 22 August 2017

(C) The Author(s) 2017. This article is an open access publication

\begin{abstract}
The main challenge in the use of multi-wall carbon nanotube (MWCNT) as key components of nanofluids is to transfer excellent thermal properties from individual nanotubes into the bulk systems. We present studies on the performance of heat transfer nanofluids based on ultra-long $(\sim 2 \mathrm{~mm})$, curly MWCNTs - in the background of various other nanoC-sp ${ }^{2}$, i.e. oxidized MWCNTs, commercially available Nanocyl ${ }^{\mathrm{TM}}$ MWCNTs and spherical carbon nanoparticles (SCNs). The nanofluids prepared via ultrasonication from water and propylene glycol were studied in terms of heat conductivity and heat transfer in a scaled up thermal circuit containing a copper helical heat exchanger. Ultra-long curly MWCNT (1 wt.\%) nanofluids (stabilized with Gum Arabic in water) emerged as the most thermally conducting ones with a $23-30 \%$ - and $39 \%$ enhancement as compared to the base-fluids for water and propylene glycol, respectively. For turbulent flows $(R e=8000$ 11,000 ), the increase of heat transfer coefficient for the overmonths stable $1 \mathrm{wt} . \%$ ultra-long MWCNT nanofluid was found as high as $>100 \%$. The findings allow to confirm that longer
\end{abstract}

Electronic supplementary material The online version of this article (doi:10.1007/s00231-017-2133-1) contains supplementary material, which is available to authorized users.

Sławomir Boncel

slawomir.boncel@polsl.pl

1 Department of Organic Chemistry, Biochemistry and Biotechnology, Silesian University of Technology, Krzywoustego 4, 44-100 Gliwice, Poland

2 Department of Chemical and Process Engineering, Silesian University of Technology, Strzody 7, 44-100 Gliwice, Poland

3 Institute of Engineering Materials and Biomaterials, Silesian University of Technology, Konarskiego 18A,

44-100 Gliwice, Poland
MWCNTs are promising solid components in nanofluids and hence to predict their broader application in heat transfer media.

\section{Introduction}

With a continuous development of power plants, solar collectors, machines, machineries, devices and advancing miniaturization of electronics as well as increasing number of supercomputers, heat transfer intensification becomes a critical phenomenon [1, 2]. The main challenges in the heat transfer processing based on nanofluids (base-fluids containing uniformly dispersed nanoparticles) are: (a) high thermal conductivity and high convective heat transfer coefficient in thermal systems enabling enhanced energy harvesting, (b) high energy conversion efficiency (e.g. between radiation energy of sunlight into the heating medium), (c) physicochemical stability over storage and working (the latter frequently under harsh conditions), (d) prevention of clogging in microchannels, (e) minimized biological and chemical corrosion of the construction materials caused e.g. by bacteria or acids formed via oxidation of base fluids like glycols, and (f) low abrasion of piping by dispersed nanoparticles [3]. Those problems have already met response from various nanomaterials (metal, metal oxides and other nanoparticles) with partial successes $[4,5]$. Among many possible, multi-wall carbon nanotubes (MWCNTs) - nanoparticles with their C-sp ${ }^{2}$ (hybridization) 1D-geometry and a unique combination of physical, chemical and biological properties - are considered as an ideal candidate for large-scale thermal applications [6-10]. This nanomaterial is now available at the industrial scale and is by orders of magnitude less expensive than its single-wall counterpart [11]. However, the critical challenge in the use of MWCNTs is 'translation' of their excellent properties from the individual nanotubes (thermal conductivity ca. $3000 \mathrm{~W} \mathrm{~m}^{-1} \mathrm{~K}^{-1}$ for MWCNT $[12,13])$ into the nanofluids (or other bulk assemblies 
such as polymer composites), where in thermal conductivity drops by orders of magnitude $[14,15]$. This obstacle is due to non-uniformity of dispersion and small interphase (contact areas) between the individual MWCNTs which can be additionally separated with (macro)molecules with lower heat conductivity. In consequence, those phenomena impede phonon transport. By now, the main area of exploitation of MWCNTs (pristine and functionalized) and other nanoC-sp ${ }^{2}$ allotropes (e.g. graphene [16]) has been fabrication of nanofluids using readily available base-fluids as water [17], oils [18] or glycols [19, 20]. From numerous studies (a cross-field review was written e.g. by Sadri et al. [21]), it is evident that MWCNT nanofluids show great promise revealing up to from 20 [22] to $72 \%$-enhancement [23] in thermal conductivity as compared to the base-fluid. In those cases, 1 wt.\% MWCNT stabilized with gum arabic (GA) in water and $0.4 \%$ wt. oxidized MWCNT in a mixture of ethylene glycol/water were used, respectively. Also, MWCNTs confirmed their potential in the heat transfer performance showing e.g. a $32 \%$-increase of convective heat transfer coefficient at $R e=500$, $\left(l_{M W C N T}=0.5-40 \mu \mathrm{m}, O D_{M W C N T}=10-20 \mathrm{~nm}, 1 \mathrm{wt} . \% \mathrm{H}_{2} \mathrm{O}\right.$, $0.25 \%$ wt. GA) [22].

Although the above indisputable results in the increase of thermal conductivity coefficients were revealed for various nanotube content and morphologies, aspect ratios, surface chemistries / surfactant stabilizers, type of basefluids and temperatures, there are inconsistencies and difficulties in correlation between nanotube morphology / chemistry and the heat transfer performance [24, 25]. In this paper, in order to investigate thermal performance of MWCNT-based nanofluids, we have selected curly and ultra-long versus short MWCNTs in the comparative studies among various nanoC-sp ${ }^{2}$-based allotropes as dispersed phases (with GA [26] as a stabilizing surfactant). The rationale behind the selection of nanoC-sp ${ }^{2}$ was twofold. Firstly, the critical parameter of heat transfer efficiency is dimensionality of the nanoparticles forming thermally conducting pathways [27]. It was therefore important to compare two MWCNT-based nanofluids where morphologies of nanotubes would be strikingly different, and also with a nanoC-sp ${ }^{2}$ allotrope of spherical structure, i.e. spherical carbon nanostructures (SCNs). This comparison was also critical as commercially available MWCNTs are typically of low aspect ratios. Additionally, hence surface chemistry is usually responsible for disintegration of the outer nanotube walls and functionalization leads to prolongation of the MWCNT dispersions stability, OMWCNTs were also used for comparison.

\section{Materials and methods}

Four different nanoC-sp ${ }^{2}$ phases were prepared as components of the heat-transfer nanofluids. Ultra-long (as well as precursors of O-MWCNTs) MWCNTs were synthesized via chemical catalytic vapour deposition (c-CVD) at $760{ }^{\circ} \mathrm{C}$ in argon using toluene and ferrocene as carbon feedstock and catalyst precursor, respectively [28]. As the second nanophase towards months-stable aqueous dispersions, O-MWCNTs were prepared by the treatment of $250 \pm 120 \mu \mathrm{m}$-long MWCNTs with a boiling mixture of $98 \% \mathrm{H}_{2} \mathrm{SO}_{4}$ and $68 \% \mathrm{HNO}_{3}(3: 1, v / v)$ according to our previous work [29]. Spherical carbon nanostructures (SCNs) produced via c-CVD from acetylene using pre-deposited and then $\mathrm{H}_{2}$-pretreated iron nanoparticles as catalysts at $800{ }^{\circ} \mathrm{C}$. Commercially available Nanocyl ${ }^{\mathrm{TM}} \mathrm{NC} 7000$ MWCNTs were tested as the last nanophase. SEM images were acquired using a Tabletop SEM HITACHI TM3000 (accelerating voltage: $15 \mathrm{kV}$, tungsten source, $30 \mathrm{~nm}$ resolution). TEM imaging was performed on JEOL 4000EX-II (LaB6 electron source, operated at $400 \mathrm{kV}$ ) and Philips Tecnai F20 operated at $200 \mathrm{kV}$ accelerating voltage. Initially, four different nanofluids containing $1 \mathrm{wt} \%$ of nanoC-sp ${ }^{2}$ were prepared, i.e. (1) MWCNTs $+0.5 \mathrm{wt} \% \mathrm{GA} /$ water, (2) O-MWCNTs in water, (3) Nanocyl ${ }^{\mathrm{TM}}$ MWCNTs $+0.5 \mathrm{wt} \% \mathrm{GA} /$ water, (4) SCNs + $1 \mathrm{wt} \% \mathrm{GA} /$ water. For further experiments, MWCNT dispersions in propylene glycol (PG) - as medium of low melting point and more environmentally friendly than ethylene glycol were also studied. 1-h ultrasonication (Sonics VCX-130, probe diameter $13 \mathrm{~mm}$ ) was used as the agitation technique prior to the measurements. Thermal conductivities of the nanoC-sp ${ }^{2}$ nanofluids at various temperatures were measured using a $\mathrm{KD}$ 2 Pro thermal properties analyzer (Decagon devices, Inc., USA) equipped with a 6-cm KS-1 probe. A principle of this measurement is Transient Hot Wire (THW) method which provides an accuracy of $\pm 5 \%$. Viscosity was measured using a viscometer Brookfield LV-II + Pro at selected temperatures which were measured with accuracy $\pm 0.1{ }^{\circ} \mathrm{C}$. Heat transfer coefficient in a helical heat exchanger (Fig. S1, A) was measured in a laboratory setup presented in Fig. 1.

Nanofluid from vessel (1) was delivered by pump (2) to the copper helical coil heat exchanger (4) (diameter of coil $96 \mathrm{~mm}$, tube inner diameter $6.2 \mathrm{~mm}$, length $L=2530 \mathrm{~mm}$ ) immersed in the insulated container (5) and filled with deionized water (DW). Average temperature of DW was kept at $60 \pm 1^{\circ} \mathrm{C}$ by means of an integrated electrical heater and stirrer (6). Wall temperature of the helical heat exchanger was measured by means of seven attached K-type thermocouples which were connected to $\mathrm{A} / \mathrm{C}$ Advantech converter. Before experiments, the thermocouples were calibrated with accuracy $\pm 0.1{ }^{\circ} \mathrm{C}$. Outlet and inlet temperatures of the nanofluid were measured by means of a separate set of K-type calibrated thermocouples. Flow rate of nanofluid was measured by flowmeter (3) Flowmex PV 40 (Codea, Czech Republic). After leaving helical exchanger, the nanofluid was cooled down in a system of the tube-shell heat exchangers (7), and then returned to the container. 
Fig. 1 Scheme of laboratory setup: 1 - fluid container, 2 pump, 3 - flowmeter, 4 - helical coil heat exchanger, 5 - insulated container, 6 - integrated heater and stirrer, 7 - cooling system

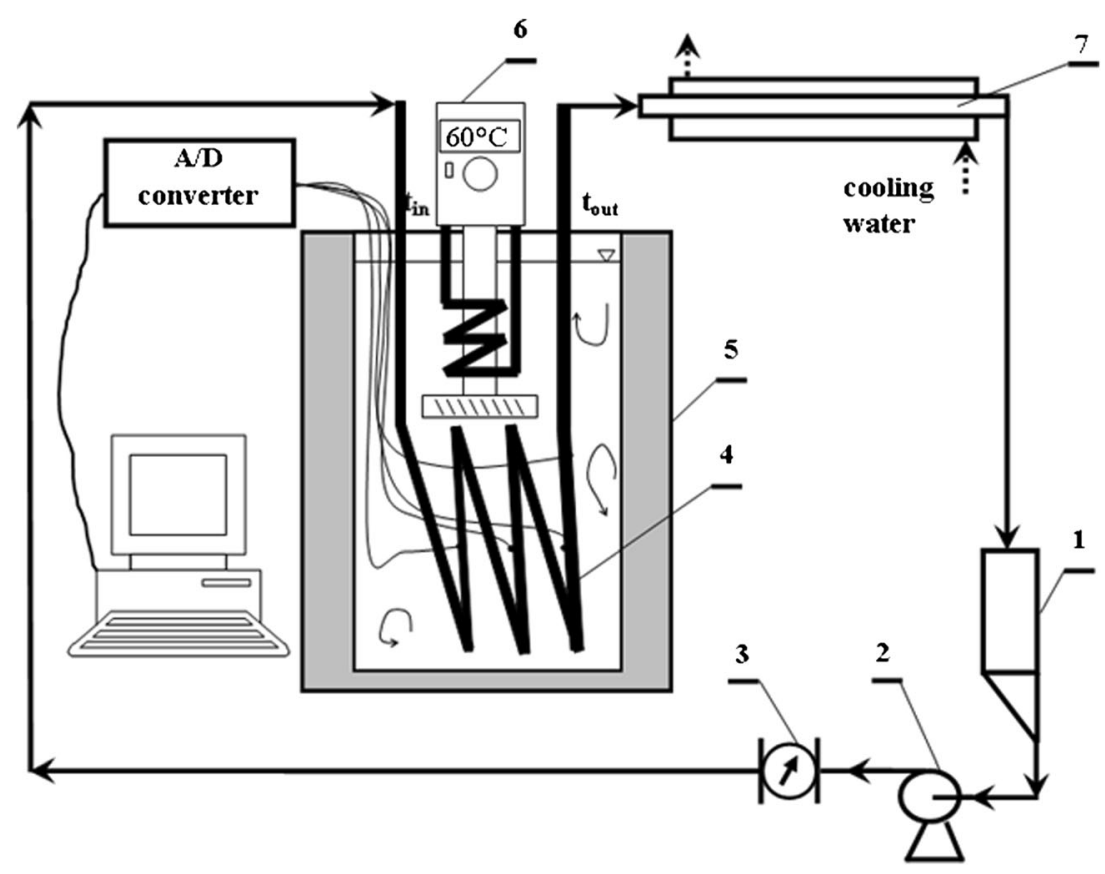

\section{Results and discussion}

Morphology of nanoC-sp ${ }^{2}$ dispersed phases is presented in SEM and TEM images (Fig. 2). As length and waviness of MWCNTs can be controlled by duration time and pressure in the c-CVD furnace, $\sim 2 \mathrm{~mm}$ and curly (outer and inner diameter of 10 and $60 \mathrm{~nm}$, respectively) MWCNTs were synthesized (aspect ratio $3 \times 10^{5}$ ) (Fig. 2a, e). The synthesis was performed according to a known procedure [28], modified by applying higher internal pressure in the CVD furnace and prolonged $(14 \mathrm{~h})$ nanotube growth. OMWCNTs were $0.8 \pm 0.4 \mu \mathrm{m}$ long and $21 \pm 11 \mathrm{~nm}$ thick (Fig. 2b, f). As MWCNTs from the other end of the aspect ratio scale - commercially available Nanocyl ${ }^{\mathrm{TM}} \mathrm{NC} 7000$ were used and their highly-entangled spaghetti-like micro-assemblies of open-tip and a few-wall structure was confirmed by SEM (Fig. 2c) and TEM (Fig. 2g). Nanocyl ${ }^{\mathrm{TM}}$ MWCNTs contain $10 \%$ of catalyst - mainly as $\mathrm{Al}_{2} \mathrm{O}_{3}$ [30]. SCNs of $20 \pm 10 \mathrm{~nm}$ in diameter revealed entangled morphology with onion-like graphitic layers surrounding catalyst nanoparticles (Fig. $2 \mathrm{~d}, \mathrm{~h}$ ) and were heavily loaded up to ca. 11 wt.\% with iron nanoparticles as measured by inductively coupled plasma atomic emission spectrometry (ICP-AES) [31].

All tested nanofluids of the identical (per weight) nanoC-sp ${ }^{2}$ allotrope content caused an increase in thermal conductivity as compared to the base-fluids in the range of temperatures $5-65{ }^{\circ} \mathrm{C}$ (Fig. 3).

Initially, water containing GA was used as the base fluid (Fig. 3a). The lowest and only ca. 5\% increase was found for commercially available Nanocyl ${ }^{\mathrm{TM}}$ MWCNTs. SCN- based nanofluid could be found as ca. 15\% more conductive on average than the base-fluid but a high drop of dispersion stability was observed even at $25^{\circ} \mathrm{C}$. Rather intense sedimentation of solid SCNs, partially connected with their high density, could by visible by naked eye and it was further confirmed as a drop in thermal conductivity. Oxidation of MWCNTs causing cutting and introduction of oxygen-rich functionalities (carboxylic, hydroxyl) as well as defects to the outer MWCNT walls resulted in the formation of months-stable dispersion of still excellent heat conductivity, ca. $12 \%$ more conducting than water. Nevertheless, 1 wt.\% ultra-long MWCNTs in water emerged as the most thermally conducting among the all tested water-based nanofluids yielding a 23-30\% enhancement as compared to water + GA system. As pure water cooling liquids are rarely applied in industry, the most promising curly ultra-long MWCNTs were selected as the appropriate dispersed phase for preparation of PG-based nanofluid. PG was selected as it is non-toxic, water-miscible, inexpensive and broadly used [32]. Here, the enhancement was even more evident - PG-based nanofluid containing ultra-long MWCNTs emerged as 39\% more conducting heat than PG. (Thermal conductivity for PG was established only up to $35^{\circ} \mathrm{C}$ due to increasingly significant convection). Moreover, in the range of $0-1$ wt.\% nanotube content, thermal conductivity was found as linearly dependent on the nanotube concentration as at $0.5 \mathrm{wt} . \%$ MWCNTs thermal conductivity of the nanofluid was $0.28 \mathrm{~W} \mathrm{~m}^{-1} \mathrm{~K}^{-1}$ on average. This finding is also in agreement with previous works showing that heat conductivity was higher for nanofluids based on longer MWCNTs [33]. 
Fig. 2 SEM (a-d) and TEM (eh) images of curly ultra-long MWCNTs, O-MWCNTs, Nanocyl ${ }^{\mathrm{TM}}$ NC7000 MWCNTs and SCNs; insets in (a) show fibrous nature of MWCNTs (left) and thickness of MWCNT forest
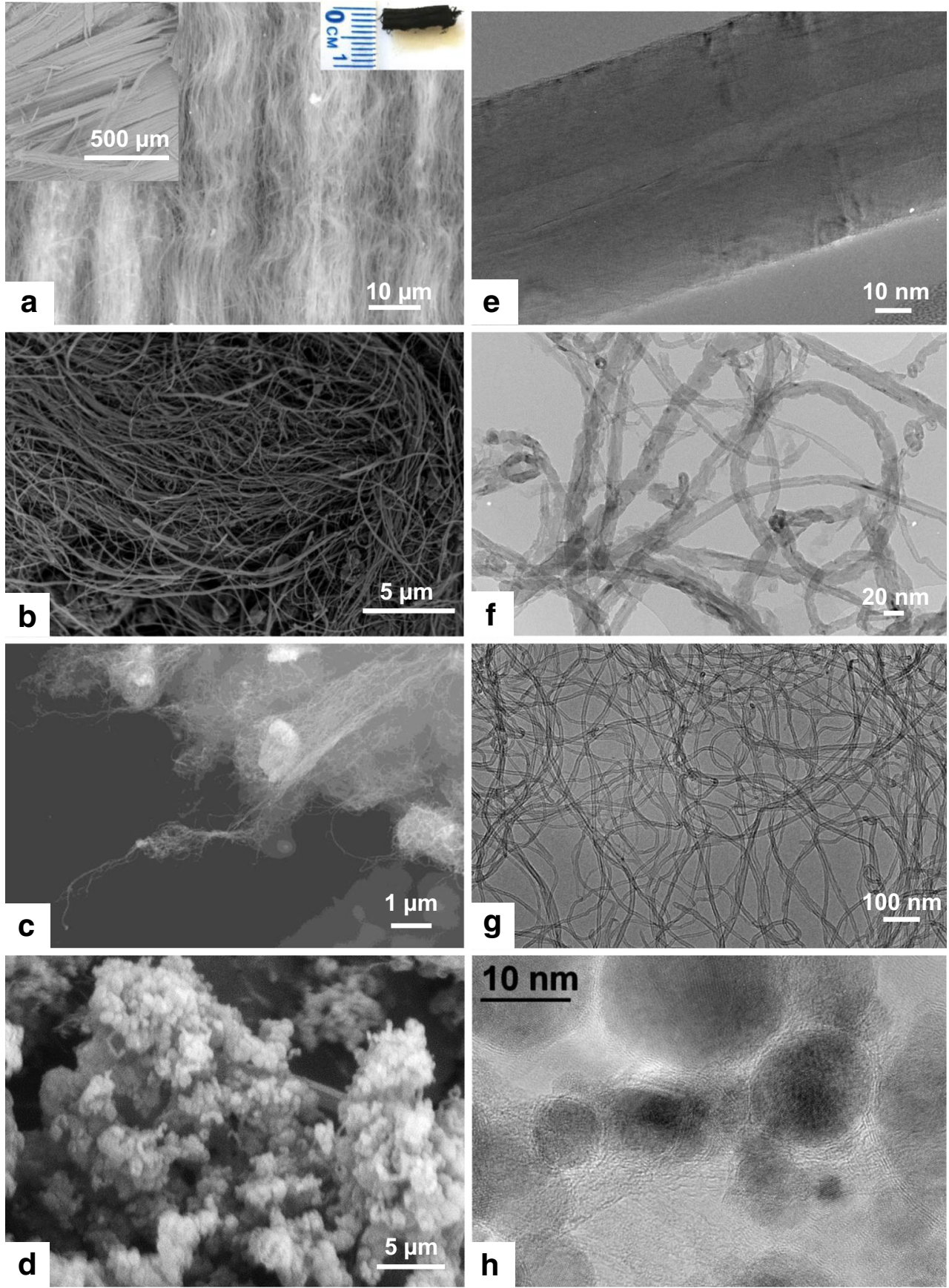

The fundamental of size reduction of the systems used in thermal engineering is the enhancement of heat transfer. For this purpose, two main techniques, i.e. active and passive are used [34]. The former one uses electric, acoustic or vibration field interaction to increase the heat transfer coefficient. In the passive technique, intensification of the heat transfer is achieved by modification of geometry of heat exchanger and/ or addition of materials to modify media that flow inside the equipment. One of the widely used passive solutions is a coil which is used in chemical (reactors, distillation columns, evaporators) and related industries (HVAC equipment, sanitary engineering, steam generators and condensers). This is due to a high value of the heat transfer surface area per unit volume. Geometry of the helical coil induces secondary flow due to unbalanced centrifugal forces and enhanced cross-sectional mixing which are responsible for the increase in the heat transfer coefficient [35]. Hence, combining coil geometry and nanofluids, as the next step, the most promising 0.5 and 1 wt $\%$ ultra-longMWCNTs water-based nanofluid stabilized with $0.5 \%$ wt. GA was examined in terms of heat transfer enhancement (Fig. 4, a detailed error analysis for those measurements is presented in SD and in SD Table 1). Viscosity of the MWCNT nanofluid was 


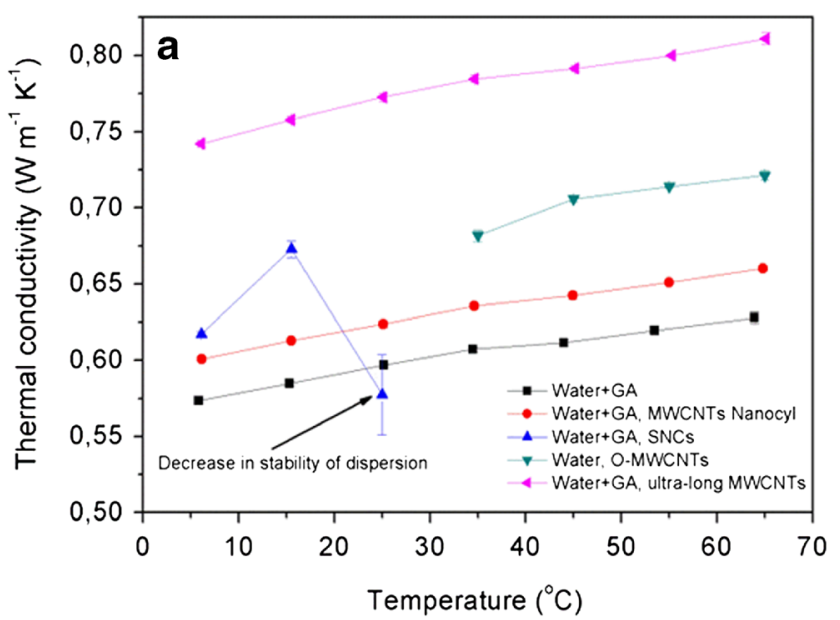

Fig. 3 a Thermal conductivity versus temperature for various nanoC-sp ${ }^{2}$ nanofluids with water as a base-fluid, b Thermal conductivity versus temperature for the ultra-long MWCNT based nanofluid as the most

found in the range of 1.7 to $1.2 \mathrm{mPa} \cdot \mathrm{s}$ for 20 and $70{ }^{\circ} \mathrm{C}$, respectively, allowing for application of a standard pump in the scaledup system. Also, the temperature-viscosity relationship fulfilled the Arrhenius Eq. (SD, Fig. S1, B).

Heat transfer coefficient $(h)$ for the examined nanofluids was calculated as average of 7 local values $h\left(x_{i}\right)$ measured along helical coil and calculated as follows:

$h\left(x_{i}\right)=\frac{q}{t_{w}\left(x_{i}\right)-t\left(x_{i}\right)}$,

where $t_{w}\left(x_{i}\right), t\left(x_{i}\right)$, are measured temperature of wall and local bulk nanofluid temperature, respectively. Local bulk temperature was estimated as:

$t\left(x_{i}\right)=\frac{t_{\text {out }}-t_{\text {in }}}{L} x_{i}+t_{\text {in }}$,

where $t_{\text {in }}$ and $t_{\text {out }}$ are measured bulk temperatures of nanofluid at the inlet and outlet, respectively, $L$ working length of helical tube and $x_{i}$ location relatively to the inlet. Heat flux $(q)$ absorbed by the fluid was calculated as follows:

$q=\frac{\dot{V} \rho_{n f} c_{n f}\left(t_{\text {out }}-t_{\text {in }}\right)}{F}$,

where: $\dot{V}$ - flow rate, $\left[\mathrm{m}^{3} / \mathrm{s}\right], c_{n f}$ - heat capacity of nanofluid, $[\mathrm{J} / \mathrm{kg} \mathrm{K}], F$ - inner surface area of heat transfer, $F=0.0493 \mathrm{~m}^{2}$. Effective heat capacity of nanofluid was calculated as [36]:

$c_{n f}=\frac{\phi_{N W} \rho_{N W} c_{N W}+\left(1-\phi_{N W}\right) \rho_{w} c_{w}}{\rho_{n f}}$,

where: subscripts 'NW' and ' $w$ ' denote ultra-long-MWCNTs and water, respectively, $\phi_{N W}$ volume fraction, $\rho_{N W}$ density of

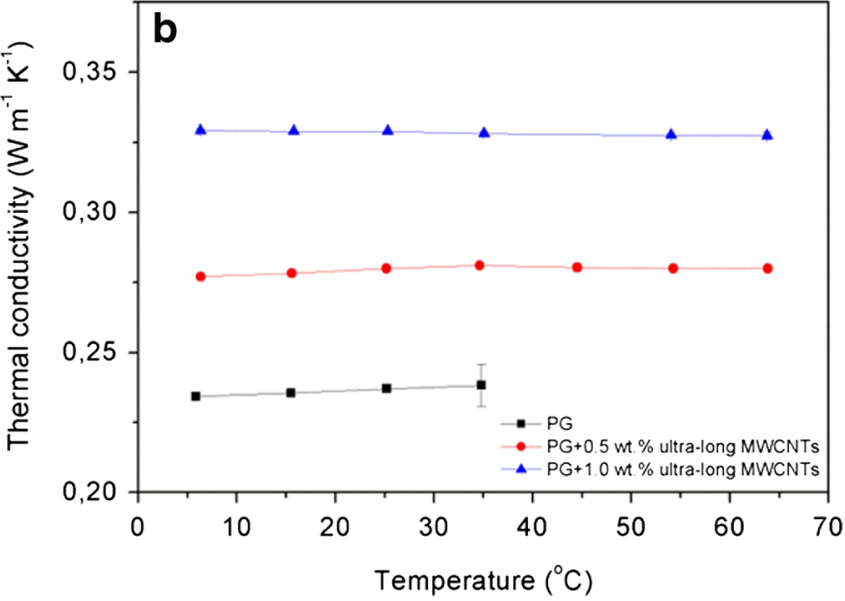

promising nanoC-sp ${ }^{2}$ system with propylene glycol (PG) as a basefluid; the lines serve only as eye-guidelines; the error bars represent standard deviations from at least four isolated measurements

solid $\left(\rho_{N W}=2100 \mathrm{~kg} / \mathrm{m}^{3}[37]\right), c_{N W}$ heat capacity of MWCNT $\left(c_{N W}=750 \mathrm{~J} / \mathrm{kgK}[38]\right)$, density of nanofluid was calculated from equation:

$\rho_{n f}=\phi_{N W} \rho_{N W}+\left(1-\phi_{N W}\right) \rho_{w}$.

Firstly, the accuracy of the method for determining of $h$ was examined using DW. Figure 3 shows comparison of average Nusselt number measured and calculated using equation (Eq. 6) [34]:

$N u=\frac{h d}{\lambda}=0.00619\left(\frac{u \rho d}{\eta}\right)^{0.92} \operatorname{Pr}^{0.4}\left(1+3.455 \frac{d}{D}\right)$.

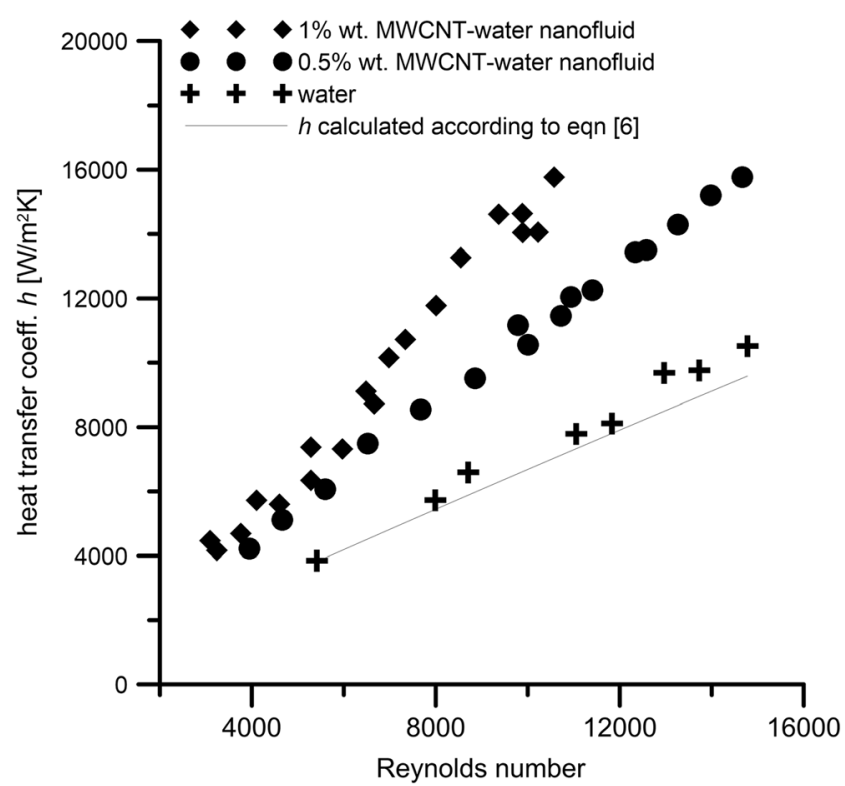

Fig. 4 Heat transfer coefficient $(h)$ versus Reynolds number $(R e)$ for the selected nanofluids based on ultra-long MWCNTs, GA and water 
The equation was obtained for developed turbulent flow for $5000<R e<100,000$. Heat conductivity coefficient $\left(\lambda_{n f}\right)$ of the nanofluids was estimated at average fluid bulk temperature. Experimentally determined $h$-values for DW were slightly higher than theoretically estimated, and the maximal discrepancy did not exceed $7 \%$ with regard to the calculated one using Eq. 6. Experiments with 0.5 and 1 wt.\% ultra-long MWCNT nanofluids showed concentration-dependent and significant enhancement of heat transfer coefficient in comparison with the results obtained with DW $-h$ increased with the solid contents in the examined nanofluids. For Reynolds number $(R e)$ in the range of 8000 to 11,000 , the increase of heat transfer coefficient for $1 \mathrm{wt} . \%$ MWCNT nanofluid was found as high as $>100 \%$. Importantly, quality of the ultra-long MWCNT nanofluid has not been changed throughout the over-month storage and showed practically equal results.

\section{Conclusions}

In the background of spherical and cylindrical nanoC-sp ${ }^{2}$ allotropes, ca. $2 \mathrm{~mm}$-long curly MWCNT-based nanofluid was found as the most promising heat transfer medium, probably due to the optimal geometry of the dispersed phase enabling formation of conducting pathways. The results showed that the presence of curly and long MWCNTs, fully dispersible in $\mathrm{GA} /$ water, enhanced heat transfer coefficient in the flow of nanofluids in helical coil in comparison with pure water. It allows us to predict that MWCNT-based nanofluids, also due to significantly higher and less expensive world production of MWCNTs, will soon find application as efficient heat transfer media.

Acknowledgements The authors are very grateful to the Foundation for Polish Science (39/UD/SKILLS/2015, 'Impuls' program) and National Science Centre in cooperation with National Science for Research and Development (program TANGO, ID = 266702) for the financial support as well as Winiplast Company (Orzesze, Poland) for technical and professional assistance as well fruitful discussions on the applications of MWCNT nanofluids.

\section{Compliance with ethical standards}

Conflict of interest On behalf of all authors, the corresponding author states that there is no conflict of interest.

Open Access This article is distributed under the terms of the Creative Commons Attribution 4.0 International License (http:// creativecommons.org/licenses/by/4.0/), which permits unrestricted use, distribution, and reproduction in any medium, provided you give appropriate credit to the original author(s) and the source, provide a link to the Creative Commons license, and indicate if changes were made.

\section{References}

1. Lienhard JH IV, Lienhard VJH (2008) A heat transfer textbook, 3rd edn. Phlogiston Press, Cambridge

2. Moore AL, Shi L (2014) Emerging challenges and materials for thermal management of electronics. Mater Today 17:163-174. doi:10.1016/j.mattod.2014.04.003

3. Lomascolo M, Colangelo G, Milanese M, de Risi A (2015) Review of heat transfer in nanofluids: conductive, convective and radiative experimental results. Renew Sust Energ Rev 43:1182-1198. doi:10. 1016/j.rser.2014.11.086

4. Angayarkanni SA, Philip J (2015) Review on thermal properties of nanofluids: recent developments. Adv Colloid Interf Sci 225:146176. doi:10.1016/j.cis.2015.08.014

5. Buongiorno J, Venerus DC, Prabhat N et al (2009) A benchmark study on the thermal conductivity of nanofluids. J Appl Phys 106: 094312. doi:10.1063/1.3245330

6. Hone J (2004) Dekker encyclopedia of nanoscience and nanotechnology. Marcel Dekker, New York, pp 603-610. doi:10.1081/EENN120009128

7. Fabris D, Rosshirt M, Cardenas C et al (2011) Application of carbon nanotubes to thermal interface materials. J Electron Packag 133:020902. doi:10.1115/1.4003864

8. Hone J, Llaguno MC, Biercuk MJ et al (2002) Thermal properties of carbon nanotubes and nanotube-based materials. Appl Phys A Mater Sci Process 74:339-343. doi:10.1007/s003390201277

9. Kaur S, Raravikar N, Helms BA et al (2014) Enhanced thermal transport at covalently functionalized carbon nanotube array interfaces. Nat Commun 3082. doi:10.1038/ncomms4082

10. Cornelio JAC, Cuervo PA, Hoyos-Palacio LM et al (2016) Tribological properties of carbon nanotubes as lubricant additive in oil and water for a wheel-rail system. J Mater Res Technol 5:6876. doi:10.1016/j.jmrt.2015.10.006

11. De Volder MFL, Tawfick SH, Baughman RH, Hart AJ (2013) Carbon nanotubes: present and future commercial applications. Science 339:535-539. doi:10.1126/science.1222453

12. Samani MK, Khosravian N, Chen GCK et al (2012) Thermal conductivity of individual multiwalled carbon nanotubes. Int J Therm Sci 62:40-43. doi:10.1016/j.ijthermalsci.2012.03.003

13. Kim P, Shi L, Majumdar A, McEuen PL (2001) Thermal transport measurements of individual multiwalled nanotubes. Phys Rev Lett 87:215502. doi:10.1103/PhysRevLett.87.215502

14. Singh IV, Tanaka M, Endo M (2007) Effect of interface on the thermal conductivity of carbon nanotube composites. Int $J$ Therm Sci 46:842-847. doi:10.1016/j.ijthermalsci.2006.11.003

15. Kwon SY, Kwon IM, Kim Y-G et al (2013) A large increase in the thermal conductivity of carbon nanotube/polymer composites produced by percolation phenomena. Carbon 55:285-290. doi:10. 1016/j.carbon.2012.12.063

16. Sadeghinezhad E, Mehrali M, Saidur R et al (2016) A comprehensive review on graphene nanofluids: recent research, development and applications. Energy Convers Manag 111: 466-487. doi:10.1016/j.enconman.2016.01.004

17. Assael MJ, Chen C-F, Metaxa I, Wakeham WA (2004) Thermal conductivity of suspensions of carbon nanotubes in water. Int $\mathrm{J}$ Thermophys 25:971-985. doi:10.1023/B:IJOT.0000038494. 22494.04

18. Hwang Y, Park HS, Lee JK, Jung WH (2006) Thermal conductivity and lubrication characteristics of nanofluids. Curr Appl Phys 6: e67-e71. doi:10.1016/j.cap.2006.01.014

19. Liu M-S, Ching-Cheng Lin M, Huang I-T, Wang C-C (2005) Enhancement of thermal conductivity with carbon nanotube for nanofluids. Int Commun Heat Mass Transf 32:1202-1210. doi: 10.1016/j.icheatmasstransfer.2005.05.005 
20. Ruan B, Jacobi AM (2012) Ultrasonication effects on thermal and rheological properties of carbon nanotube suspensions. Nanoscale Res Lett 7:127. doi:10.1186/1556-276X-7-127

21. Sadri R, Ahmadi G, Togun H et al (2014) An experimental study on thermal conductivity and viscosity of nanofluids containing carbon nanotubes. Nanoscale Res Lett 9:151-166. doi:10.1186/1556276X-9-151

22. Garg P, Alvarado JL, Marsh C et al (2009) An experimental study on the effect of ultrasonication on viscosity and heat transfer performance of multi-wall carbon nanotube-based aqueous nanofluids. Int J Heat Mass Transf. doi:10.1016/j.ijheatmasstransfer.2009.04.029

23. Singh N, Chand G, Kanagaraj S (2012) Investigation of thermal conductivity and viscosity of carbon nanotubes-ethylene glycol nanofluids. Heat Transf Eng 33:821-827. doi:10.1080/01457632. 2012.646922

24. Murshed SMS, Nieto de Castro CA (2014) Superior thermal features of carbon nanotubes-based nanofluids - a review. Renew Sust Energ Rev 37:155-167. doi:10.1016/j.rser.2014.05.017

25. Nagarajan PK, Subramani J, Suyambazhahan S, Sathyamurthy R (2014) Nanofluids for solar collector applications: a review. Energy Procedia 61:2416-2434. doi:10.1016/j.egypro.2014.12.017

26. Bandyopadhyaya R, Nativ-Roth E, Regev O, Rozen RY (2001) Utilizing old Egyptian wisdom for stabilization of individual carbon nanotubes in aqueous dispersions. MRS Proc 706:Z9.28.1. doi:10. 1557/PROC-706-Z9.28.1

27. Chopkar M, Sudarshan S, Das PK, Manna I (2008) Effect of particle size on thermal conductivity of nanofluid. Metall Mater Trans A 39:1535-1542. doi:10.1007/s11661-007-9444-7

28. Boncel S, Pattinson SW, Geiser Vet al (2014) En route to controlled catalytic CVD synthesis of densely packed and vertically aligned nitrogen-doped carbon nanotube arrays. Beilstein J Nanotechnol 5: 219-233. doi:10.3762/bjnano.5.24

29. Boncel S, Müller KH, Skepper JN et al (2011) Tunable chemistry and morphology of multi-wall carbon nanotubes as a route to non- toxic, theranostic systems. Biomaterials 32:7677-7686. doi:10. 1016/j.biomaterials.2011.06.055

30. White CM, Banks R, Hamerton I, Watts JF (2016) Characterisation of commercially CVD grown multi-walled carbon nanotubes for paint applications. Prog Org Coatings 90:44-53. doi:10.1016/j. porgcoat.2015.09.020

31. Bok-Badura J, Jakóbik-Kolon A, Turek M et al (2015) A versatile method for direct determination of iron content in multiwall carbon nanotubes by inductively coupled plasma atomic emission spectrometry with slurry sample introduction. RSC Adv 5:101634-101640. doi:10.1039/C5RA22269A

32. Nelson WM (2003) Green solvents for chemistry: perspectives and practice, green chemistry series. Oxford University Press, Oxford

33. Jiang W, Ding G, Peng H (2009) Measurement and model on thermal conductivities of carbon nanotube nanorefrigerants. Int $\mathrm{J}$ Therm Sci 48:1108-1115. doi:10.1016/j.ijthermalsci.2008.11.012

34. Naphon P, Wongwises S (2006) A review of flow and heat transfer characteristics in curved tubes. Renew Sust Energ Rev 10:463-490. doi:10.1016/j.rser.2004.09.014

35. Vashisth S, Kumar V, Nigam KDP (2008) A review on the potential applications of curved geometries in process industry. Ind Eng Chem Res 47:3291-3337. doi:10.1021/IE701760H

36. Pak BC, Cho YI (1998) Hydrodynamic and heat transfer study of dispersed fluids with submicron metallic oxide particles. Exp Heat Transf 11:151-170. doi:10.1080/08916159808946559

37. Shanbedi M, Amiri A, Heris SZ, Kazi SN (2015) Nanofluids basic principles and modern aspects. In: Ildusovich Kharisov B, Vasilievna Kharissova O, Ortiz-Mendez U (eds) CRC concise encyclopedia of nanotechnology (pp 724-768). CRC Press, Boca Raton

38. Pradhan NR, Duan H, Liang J, Iannacchione GS (2009) The specific heat and effective thermal conductivity of composites containing single-wall and multi-wall carbon nanotubes. Nanotechnology 20:245705. doi:10.1088/0957-4484/20/24/245705 